\title{
LEVEL SETS FOR FUNCTIONS CONVEX IN ONE DIRECTION
}

\author{
JOHNNY E. BROWN
}

\begin{abstract}
Goodman and Saff conjectured that if $f$ is convex in the direction of the imaginary axis then so are the functions $\frac{1}{r} f(r z)$ for all $0<r<\sqrt{2}-1$, i.e., the level sets $f(|z|<r)$ are convex in the direction of the imaginary axis for $0<r<\sqrt{2}-1$. A weak form of this conjecture is proved and a question of Brannan is answered negatively.
\end{abstract}

Let $U_{r}=\{z:|z|<r\}$ and let $S$ denote the class of all functions $f(z)=z+a_{2} z^{2}$ $+\cdots$ analytic and univalent in $U=U_{1}$. For $f \in S$ there are several geometric properties possessed by $f(U)$ that are inherited by its level sets $G_{r} \equiv f\left(U_{r}\right)$ for all $0<r<1$. For example if $f(U)$ is either convex, starlike, or close-to-convex, then so are its level sets $G_{r}$ for all $0<r<1$.

An analytic function $f$ is said to be convex in the direction of a line $L_{\theta}: t e^{i \theta}$ $(-\infty<t<\infty)$ if the intersection of $f(U)$ with each line parallel to $L_{\theta}$ is either a connected set or empty. Let CIA denote those functions $f$ for which $f(U)$ is convex in the direction of the imaginary axis with $f(0)=0$ and $f^{\prime}(0)=1$. Since CIA functions are close-to-convex, they are univalent. It came as a bit of a surprise when Hengartner and Schober [4] constructed an example where $f \in C I A$ but the corresponding level sets $G_{r}$ were not convex in the direction of the imaginary axis for all $r$ sufficiently close to 1 . A more quantitative result was obtained by Goodman and Saff [3]. They were able to prove that for each $\sqrt{2}-1<r<1$ there exists an $f \in C I A$ for which $\frac{1}{r} f(r z) \notin C I A$. Hence they conjectured that if $f \in C I A$ then $\frac{1}{r} f(r z) \in C I A$ for all $0<r<\sqrt{2}-1$, i.e., the level sets $G_{r}$ are convex in the direction of the imaginary axis for $0<r<\sqrt{2}-1$.

In [1, Problem 6.53] Brannan asked whether or not: If $f \in C I A$ and $\frac{1}{r_{0}} f\left(r_{0} z\right) \notin C I A$ for some $0<r_{0}<1$, does this imply that $\frac{1}{r} f(r z) \notin C I A$ for all $r_{0}<r<1$ ? This question was motivated by the example constructed by Hengartner and Schober [4]. In this note we prove a weaker form of the Goodman-Saff conjecture and answer Brannan's question. Our main result is the following theorem.

THEOREM. If $f \in C I A$ then there exists a set $I \subseteq[0, \pi]$ of positive measure such that $\frac{1}{r} f(r z)$ is convex in the directions $L_{\theta}: t e^{i \theta}(-\infty<t<\infty)$ for all $0<r<\sqrt{2}-1$ and all $\theta \in I$.

Received by the editors March 27, 1986.

1980 Mathematics Subject Classification (1985 Revision). Primary 30C45; Secondary 30C20. 
The proof of this theorem depends on a representation formula for CIA functions and a result relating the total variation of the argument along a curve to the number of times the curve intersects lines through the origin.

Lemma A (Royster AND Ziegler [8]). A function $f$ belongs to CIA if and only if

$$
\operatorname{Re}\left\{\frac{-i z f^{\prime}(z)}{h_{\nu}\left(e^{-i \mu} z\right)}\right\}>0, \quad z \in U
$$

for some $0 \leqslant \mu, \nu \leqslant \pi$ where $h_{\nu}(z)=z /\left[1-(2 \cos \nu) z+z^{2}\right]$.

A function $f$ analytic in $U$ is said to be starlike in the direction $L_{\theta}$ : $t e^{i \theta}$ $(-\infty<t<\infty)$ if the intersection of $f(U)$ with $L_{\theta}$ is a single segment, half-line, or $L_{\theta}$. The following result gives a relation between these functions and functions convex in one direction. Our formulation follows easily from the results in [7].

LEMMA B (ROBERTSON [7]). If $z f^{\prime}(z)$ is starlike in the direction $L_{\theta}: t e^{i \theta}(-\infty<t<$ $\infty)$ for $|z|<r$, then $f(z)$ is convex in the direction $L_{\theta+\pi / 2}$ for $|z|<r$.

Finally we need a counting result given in [9] and given as a problem in [2, p. 215].

LEMMA C. If $\phi(z)$ is analytic in $|z| \leqslant r$ and $0 \notin \phi(|z|=r)$, then

$$
\int_{0}^{2 \pi}\left|\operatorname{Re}\left\{\frac{z \phi^{\prime}(z)}{\phi(z)}\right\}\right| d \theta=\int_{0}^{\pi} n(\psi) d \psi, \quad|z|=r,
$$

where $n(\psi)$ is the number of times the line te $e^{i \psi}(-\infty<t<\infty)$ intersects the curve $\phi(|z|=r)$.

Although this result is geometrically obvious, for completeness sake we give a proof using the Banach indicatrix.

Proof of Lemma C. By replacing $\phi(z)$ by $\phi\left(e^{i \alpha} z\right)$, if necessary, we may assume $\phi(r)>0$. Let $0=\theta_{1}<\theta_{2}<\cdots<\theta_{n-1}<\theta_{n}=2 \pi$ be all those angles such that $\phi\left(r e^{i \theta_{k}}\right)>0$ and $\operatorname{Arg} \phi\left(r e^{i \theta}\right)$ varies continuously from 0 to $2 \pi$ for $\theta_{k} \leqslant \theta \leqslant \theta_{k+1}$ for each $1 \leqslant k \leqslant n-1$. Note that if the curve $\phi(|z|=r)$ winds around the origin more than once, then $n \geqslant 3$. For each interval $\theta_{k} \leqslant \theta \leqslant \theta_{k+1}, 1 \leqslant k \leqslant n-1$, let $H_{k}(\theta)$ $\equiv \operatorname{Arg} \phi\left(r e^{i \theta}\right)$. Hence $0 \leqslant H_{k}(\theta) \leqslant 2 \pi$. Let $m_{k}(\psi)$ be equal to the number of roots of the equation $H_{k}(\theta)=\psi, 0 \leqslant \psi \leqslant 2 \pi$. The function $m_{k}$ is the Banach indicatrix of the function $H_{k}$ and thus

$$
\int_{0}^{2 \pi} m_{k}(\psi) d \psi=\int_{\theta_{k}}^{\theta_{k+1}}\left|d H_{k}(\theta)\right|, \quad 1 \leqslant k \leqslant n-1
$$

(see Natanson [6, p. 225] for example). Now since $\left|d H_{k}(\theta)\right|=\left|\operatorname{Re}\left\{z \phi^{\prime}(z) / \phi(z)\right\}\right| d \theta$, it follows that

$$
\int_{0}^{2 \pi} m(\psi) d \psi=\int_{0}^{2 \pi}\left|\operatorname{Re}\left\{\frac{z \phi^{\prime}(z)}{\phi(z)}\right\}\right| d \theta
$$


where $m(\psi)=m_{1}(\psi)+m_{2}(\psi)+\cdots+m_{n-1}(\psi)$ is the number of times the curve $\phi(|z|=r)$ intersects the ray $t e^{i \psi}(0<t<\infty)$ counting multiplities. Finally observe that

$$
\int_{0}^{2 \pi} m(\psi) d \psi=\int_{0}^{\pi} m(\psi)+m(\psi+\pi) d \psi=\int_{0}^{\pi} n(\psi) d \psi
$$

and so the proof of the lemma is complete.

If $\phi(z)$ is analytic and vanishes only for $z=0$ in $|z| \leqslant r$ then we assert that $n(\psi)$ is a positive even integer function except for finitely many $\psi$. To see this, suppose that $n(\psi)$ is odd for infinitely many $\left|z_{k}\right|=r$. Since $\phi(|z|=r)$ is an analytic Jordan curve surrounding the origin it follows that $\operatorname{Re}\left\{z_{k} \phi^{\prime}\left(z_{k}\right) / \phi\left(z_{k}\right)\right\}=0$. The function $\omega(z)=z \phi^{\prime}(z) / \phi(z)$ is analytic in $|z| \leqslant r$ and the curve $\omega(|z|=r)$ meets the imaginary axis an infinite number of times. Hence we can conclude that $\omega(z) \equiv i \lambda$ for some $\lambda \in \mathbf{R}$, but since $\omega(0)=1$ a contradiction is reached. The assertion is proved.

Proof of Theorem. Fix $0<r<\sqrt{2}-1$. Observe first that

$$
Q(\zeta) \equiv \operatorname{Re}\left\{\frac{\zeta h_{\nu}^{\prime}(\zeta)}{h_{\nu}(\zeta)}\right\}=\operatorname{Re}\left\{\frac{1-\zeta^{2}}{1-(2 \cos \nu) \zeta+\zeta^{2}}\right\}
$$

where $h_{\nu}$ is given in Lemma A, is harmonic in $U, Q(0)=1$, and $Q(\zeta)>0$. If $p(z)$ is analytic in $U, p(0)=1$, and $\operatorname{Re} p(z)>0$, then the following estimate was given by Libera [5]:

$$
\left|\frac{z p^{\prime}(z) \cdot}{p(z)+i \beta}\right| \leqslant \frac{2 r}{1-r^{2}}, \quad|z|=r
$$

for any $\beta \in \mathbf{R}$.

Let $f \in C I A$. Then by Lemma A we get

$$
z f^{\prime}(z)=h_{\nu}\left(e^{-i \mu} z\right)[\cos \mu+i p(z) \sin \mu]
$$

for some $0 \leqslant \mu, \nu \leqslant \pi$ and some function $p$ analytic in $U$ with $p(0)=1, \operatorname{Re} p>0$. If $\phi(z)=z f^{\prime}(z)$ then from (2) we obtain

$$
\frac{z \phi^{\prime}(z)}{\phi(z)}=\left\{\frac{\left(e^{-i \mu} z\right) h_{\nu}^{\prime}\left(e^{i \mu} z\right)}{h_{\nu}\left(e^{-i \mu} z\right)}\right\}+\left\{\frac{z p^{\prime}(z)}{p(z)-i \cot \mu}\right\}
$$

(if $\mu=0$ or $\pi$ the last term is not present). It follows from (3) and (1) that

$$
\begin{aligned}
\int_{0}^{2 \pi}\left|\operatorname{Re} \frac{z \phi^{\prime}(z)}{\phi(z)}\right| d \theta & \leqslant \int_{0}^{2 \pi} \operatorname{Re}\left\{\frac{\left(e^{-i \mu} z\right) h_{\nu}^{\prime}\left(e^{-\mu} z\right)}{h_{\nu}\left(e^{-i \mu} z\right)}\right\} d \theta+\int_{0}^{2 \pi}\left|\frac{z p^{\prime}(z)}{p(z)-i \cot \mu}\right| d \theta \\
& \leqslant 2 \pi+2 \pi\left(\frac{2 r}{1-r^{2}}\right)=2 \pi\left(\frac{1+2 r-r^{2}}{1-r^{2}}\right),
\end{aligned}
$$

where $|z|=r$. By hypothesis, $r<\sqrt{2}-1$ and hence

$$
\int_{0}^{2 \pi}\left|\operatorname{Re}\left\{\frac{z \phi^{\prime}(z)}{\phi(z)}\right\}\right| d \theta<4 \pi
$$


Now from Lemma $\mathrm{C}$ we get

$$
\int_{0}^{\pi} n(\psi) d \psi<4 \pi
$$

As pointed out earlier, since $\phi(z)=z f^{\prime}(z)$ is analytic in $|z| \leqslant r$ and vanishes only at $z=0, n(\psi) \geqslant 2$ and $n(\psi)$ is a positive even integer function except for a finite set of $\psi$, say $E_{0}=\left\{\psi_{1}, \psi_{2}, \ldots, \psi_{n}\right\}$. The inequality (4) thus gives $n(\psi)<4$ for all $\psi \in J$ for some set $J \subseteq[0, \pi]$ with positive measure. Hence $n\left(\psi^{*}\right)=2$ for all $\psi^{*} \in J \backslash E_{0} \equiv I^{*}$ and so the function $\phi(z)=z f^{\prime}(z)$ is starlike in the directions $L_{\psi^{*}}$ for all $\psi^{*} \in I^{*}$. We now apply Lemma B to conclude that for each such direction $L_{\psi^{*}}$, the function $f(z)$ is convex in the direction $L_{\psi}$, where $\psi=\psi^{*}+\pi / 2$ for $|z| \leqslant r$. This completes the proof of the theorem.

We now turn to the question of Brannan. Specifically we find a function $F \in C I A$ with $\frac{1}{r_{0}} F\left(r_{0} z\right) \notin C I A$ but $\frac{1}{r_{1}} F\left(r_{1} z\right) \in C I A$ for some $r_{0}<r_{1}<1$. The function

$$
F(z)=\frac{z-A z^{2}}{(1-B z)^{2}},
$$

where $A=e^{2 i \alpha} \cos \alpha$ and $B=e^{i \alpha}$, belongs to CIA and maps $U$ onto the exterior of a vertical slit lying along the line $\operatorname{Re} w=-\cos \alpha / 2$ (see [3]). If $(\partial / \partial \theta) \operatorname{Re} F\left(r e^{i \theta}\right)$ has exactly two sign changes in $[0,2 \pi)$, then $\frac{1}{r} F(r z) \in C I A$; while if it has four sign changes then $\frac{1}{r} F(r z) \notin C I A$. Following [3] we see that by replacing $z$ by $z e^{-i \alpha}$, the number of sign changes of $(\partial / \partial \theta) \operatorname{Re} F\left(r e^{i \theta}\right)$ is the same as the number of sign changes of $Q(\theta)$ in $[0,2 \pi)$, where

$$
Q(\theta) \equiv\left(1+r^{2}\right) \sin (\theta-\alpha)+r[3 \sin \alpha-\sin (2 \theta+\alpha)]
$$

In what follows, let $\alpha=2.6$. Suppose first that $r=r_{0}=0.5$. Then since $Q(0)=$ $Q(2 \pi)=-0.1288 \ldots, Q(1)=0.0206 \ldots, Q(2)=-0.0883 \ldots$, and $Q(\pi)=$ $1.1598 \ldots$ we can conclude that $\frac{1}{r_{0}} F\left(r_{0} z\right) \notin C I A$.

Suppose next that $r=r_{1}=0.7$. Table 1 contains $Q$ and $Q^{\prime}$ correct to six and three decimal places, respectively. Note that since $Q$ and $Q^{\prime}$ have at most four zeros in $[0,2 \pi)$, we see that $Q$ must have a zero in the interval $(0,1)$, and in $(4.2,2 \pi)$ and possibly in the interval $(2.05,2.06)$. It is easy to check that for $2.05<\theta<2.06$ we get

$$
Q(\theta)>(1.49)(-0.523)+0.7[3(0.515)-0.424]>0.005 \text {. }
$$

Hence $Q$ has exactly two zeros in $[0,2 \pi)$ and so $\frac{1}{r_{1}} F\left(r_{1} z\right) \in C I A$ and $r_{1}>r_{0}$.

\begin{tabular}{r|r|r}
\multicolumn{3}{|c}{ TABLE 1 } \\
\hline 0 & \multicolumn{1}{c}{$Q(\theta)$} & \multicolumn{1}{c}{$Q^{\prime}(\theta)$} \\
\hline 1 & -0.046391 & -0.077 \\
2.05 & 0.288771 & 0.113 \\
2.06 & 0.020353 & -0.009 \\
4.2 & 0.020351 & 0.009 \\
$2 \pi$ & 3.271910 & -0.049 \\
& -0.046391 & -0.077
\end{tabular}


Finally, it should be pointed out that our proof of the theorem cannot yield the Goodman-Saff conjecture directly as it is not sensitive to direction. We have proved the weaker conjecture that if $f$ is convex in one direction then $\frac{1}{r} f(r z)$ is convex in one direction for all $0<r<\sqrt{2}-1$. If we could rotate $C I A$ functions then the Goodman-Saff conjecture would follow immediately. The classes of convex, starlike, and close-to-convex functions are rotationally invariant, but the class of $C I A$ functions clearly is not. Despite this drawback, we believe the Goodman-Saff conjecture is true.

The author wishes to thank Y. J. Leung and Glenn Schober for a helpful conversation.

\section{REFERENCES}

1. J. M. Anderson, K. F. Barth, and D. A. Brannan, Research problems in complex analysis, Bull. London Math. Soc. 9 (1977), 129-162.

2. A. W. Goodman, Univalent functions, Mariner, Tampa, Fla., 1983.

3. A. W. Goodman and E. B. Saff, On univalent functions convex in one direction, Proc. Amer. Math. Soc. 73 (1979), 183-187.

4. W. Hengartner and G. Schober, A remark on level curves for domains convex in one direction, Applicable Anal. 3 (1973), 101-106.

5. R. Libera, Some radius of convexity problems, Duke Math. J. 31 (1964), 143-150.

6. I. P. Natanson, Theory of functions of a real variable, vol. I, Ungar, New York, 1964.

7. M. S. Robertson, Analytic functions star-like in one direction, Amer. J. Math. 58 (1936), 465-472.

8. W. C. Royster and M. Ziegler, Univalent functions convex in one direction, Publ. Math. Debrecen 23 (1976), 339-345.

9. T. Umezawa, Analytic functions convex in one direction, J. Math. Soc. Japan 4 (1952), 194-202.

Department of Mathematics, PURdue University, West LafayetTe, Indiana 47907 